\title{
NUTRIENTES DIGERIBLES, ENERGÍA NETA Y FRACCIONES PROTEICAS DE LA MORERA (Morus alba) APROVECHABLES EN VACAS LECHERAS1
}

\author{
Carlos Boschini-Figueroa ${ }^{2}$
}

\begin{abstract}
RESUMEN
Nutrientes digeribles, energía neta y fracciones proteicas de la morera (Morus alba) aprovechables en vacas lecheras. Se determinó la degradación ruminal de la materia seca, proteína cruda, extracto etéreo y fibra neutro detergente en rebrotes de morera a 70, 84, 98 y 112 días de edad. Cada una mostró propiedades propias de la edad tanto en la fracción soluble como en la velocidad de degradación. Con base en la degradabilidad a 48 horas de incubación ruminal, biológicamente se cuantificó el contenido de proteína cruda, extracto etéreo y fibra neutro detergente verdaderamente degradable. Con esos valores se determinó el contenido de nutrientes totales y la concentración de energía digestible. La concentración de nutrientes digestibles fue $56 \%$ a 70 días y $52-53 \%$ en las edades superiores, la energía digestible fue 2,57, 2,45, 2,39 y 2,34 Mcals $/ \mathrm{kg}$ de materia seca en la planta entera. La energía neta para producción de leche fue 1,22, 1,14, 1,09 y 1,06 Mcals/kg en la planta entera a los 70, 84, 98 y 112 días de rebrote, respectivamente. La fracción de proteína soluble (A) fue $37,5 \%$ a 70 días, $34-35 \%$ en las subsecuentes edades, con una fracción degradable (B) de 58,9, 59,4, 60,7 y $61,9 \%$ a $70,84,98$ y 112 días de rebrote, junto con una fracción insoluble (C) de 3,6, 4,8, 4,1 y 3,5\% respectivamente. La proteína degradada por 48 horas en el rumen fue de $90,87,83$ y $80 \%$, con una velocidad de degradación de $10,6,8,9,8,8$ y $8,7 \% / \mathrm{h}$ en orden ascendente de edad. La proteína degradable ruminalmente degradable fue $77 \%$ a 70 días y cercana a $74 \%$ en las otras edades de rebrote indicadas.
\end{abstract}

Palabras clave: Morera, energía neta, fracciones proteicas, nutrientes digestibles, edades de rebrote, ganado lechero.

\begin{abstract}
Digestible nutrients, net energy and protein fractions of mulberry fodder (Morus alba), available for dairy cows. The kinetics of ruminal degradation of dry matter, crude protein, ether extract and neutral detergent fiber was measured at four mulberry re-growth ages: 70, 84, 98 and 112 days. Each category showed characteristics corresponding to the age, not only in the soluble fraction, but also in the rate of ruminal degradability of the potentially degradable fraction. Based on the results of degradation after 48 hours of ruminal incubation, the true digestible contents of the protein and the digested ether extract and the neutral detergent fiber were determined biologically. The measurement of total digestible nutrients, and of the concentration and net rate of digestible energy were conducted as well. The concentrations of total digestible nutrients were $56 \%$ at 70 days re-growth, and 5253 at the following ages, with a digestible energy equivalent to $2.57,2.45,2.39$ and $2,34 \mathrm{Mcals} / \mathrm{kg}$ dry matter in the whole plant. Net energy for milk production was $1.22,1.14,1.09$ and $1.06 \mathrm{Mcal} / \mathrm{kg}$ in the whole plant, for 70, 84, 98 and 112 days of re-growth, respectively. The fraction of soluble protein (A) was $37.5 \%$ at 70 days, $34-35 \%$ at subsequent ages, with a degradable fraction (B) of 58.9, 59.4, 60.7 and $61.9 \%$ at $70,84,98$ and 112 re-growth days, along with an insoluble fraction (C) of 3.6, 4.8, 4.1 and $3.5 \%$ respectively. The 48 hours degraded protein in the rumen was of $90,87,83$ and $80 \%$, with degradation velocities of $10.6,8.9,8.8$ and $8.7 \% / \mathrm{h}$ in the order indicated above. The estimated rumen degradable protein was $77 \%$ at 70 days and close to $74 \%$ for the other re-growth ages.
\end{abstract}

Keywords: Mulberry, net energy, protein fractions, digestible nutrients, re-growth ages, dairy cattle.

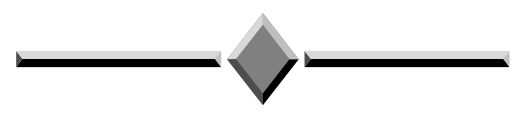

1 Recibido: 31 de octubre, 2005. Aceptado: 1 de febrero, 2006. Inscrito en la Vicerrectoría de Investigación, No. 737-A2-133.

2 Estación Experimental Alfredo Volio Mata. Facultad de Ciencias Agroalimentarias. Universidad de Costa Rica. Correo electrónico: boschini@cariari.ucr.ac.cr. 


\section{INTRODUCCIÓN}

La producción de forrajes arbóreos está siendo estudiada con intensidad, especialmente como un complemento a la dieta de rumiantes (Sayo 2000; Singh y Makkar 2000). Particularmente, en el área mesoamericana es importante la búsqueda y evaluación de nuevos cultivos forrajeros para la alimentación de vacas de alta producción lechera que requieren dietas totales, con altas concentraciones de energía y proteína (NRC 1989).

En Costa Rica, la morera muestra rendimientos superiores a $30 \mathrm{t} / \mathrm{ha} / \mathrm{año}$ de materia seca y una calidad bromatológica excepcional, con concentraciones de proteína cruda foliar superiores al $20 \%$, digestibilidad in vitro de la materia seca de $79 \%$ y de $89 \%$ para la proteína cruda (Benavides et al. 1994; Boschini et al. 1999).

La producción de leche en vacas alimentadas con morera, muestran similares rendimientos a los obtenidos con suplementación de concentrados comerciales (Boschini 2003; Oviedo 1995). A pesar de los buenos resultados mostrados, se observan variaciones importantes en la concentración de energía y proteína contenida en las distintas edades de rebrote, así como diferencias en las características bromatológicas de los forrajes de morera ofrecidos a los animales. Hasta ahora, con los resultados obtenidos, se tiene por cierto que el forraje de morera adicionado a las vacas lecheras tiene un efecto de suplementación favorable, independientemente de la edad de rebrote. Sin embargo, estas evaluaciones resultan insuficientes para llevar a cabo una adición racional y precisa de la morera como parte del componente forrajero en una ración balanceada total, siguiendo las más recientes recomendaciones del National Research Council (NRC 2001). Hasta ahora no se han reportado valores de energía neta, ni el fraccionamiento de la proteína cruda de la morera.

El presente trabajo tuvo como objetivo determinar: los nutrientes digestibles, la energía neta y fracciones de proteína contenidas en la morera, en cuatro edades de rebrote, que son fraccionados y aprovechados en vacas lecheras mediante la degradación ruminal.

\section{MATERIALES Y MÉTODOS}

El estudio se llevó a cabo en la Estación Experimental "Alfredo Volio Mata" de la Universidad de
Costa Rica, ubicada en la provincia de Cartago. Las muestras de morera se extrajeron de un lote establecido hace cuatro años. A partir de mayo del 2004 se inició el experimento con un corte de uniformización, a $60 \mathrm{~cm}$ sobre el nivel de suelo. Se empleó un diseño irrestrictamente al azar con cuatro tratamientos: 70, 84, 98 y 112 días de rebrote y seis repeticiones por tratamiento, distribuidos aleatoriamente. Un mes posterior a la uniformización, todas las parcelas fueron fertilizadas con nitrato de amonio en un equivalente de $250 \mathrm{~kg} / \mathrm{ha} /$ año de nitrógeno. La parcela experimental estuvo constituida por siete surcos con $70 \mathrm{~cm}$ de distancia por $20 \mathrm{~m}$ de largo.

$\mathrm{Al}$ cabo de cada periodo experimental de rebrote, las parcelas fueron cosechadas totalmente a la misma altura del corte de uniformización, de donde se extrajo una muestra bruta de $100 \mathrm{~kg}$ de plantas enteras para la posterior separación de tallos y hojas, las cuales fueron pesadas en fresco. De cada parte de la planta se extrajo una muestra ponderada mínima de $10 \mathrm{~kg}$, para el respectivo secado, molido a un de tamaño de partícula para los posteriores análisis de laboratorio y a $2 \mathrm{~mm}$ para la degradación ruminal. Cada muestra fue sometida al análisis de: materia seca, proteína cruda, extracto etéreo, fibra cruda, y cenizas siguiendo los métodos aprobados por el AOAC (2002).

La determinación de fibra neutro detergente, ácido detergente y lignina fueron efectuados siguiendo los procedimientos de Goering y Van Soest (1970). Adicionalmente, se analizó la proteína adherida a las fibras neutro detergente usando el método Kjeldahl (AOAC 2002). Posteriormente, fueron preparadas para el proceso de degradación ruminal, siguiendo la técnica descrita por Orskov (1984) en bolsas de nylon. Se usaron bolsas Ankon ${ }^{\circledR}$ de 10 x $20 \mathrm{~cm}$ con un poro de $53 \mu \mathrm{m}$ de diámetro, conteniendo cada una cinco gramos de muestra secada previamente a $105^{\circ} \mathrm{C}$. Las bolsas de nylon fueron suspendidas en el rumen de dos vacas fistuladas ruminalmente, mantenidas con una dieta forrajera de pasto Estrella Africana (Cynodon nlemfuensis). Las bolsas divididas en cuatro repeticiones con: $18 ; 12 ; 6 ; 6,5$ y tres observaciones por repetición en cada tiempo de incubación, introducidas consecutivamente en el rumen y mantenidas por 48 , $24,12,6$ y 2 h, respectivamente. La desaparición a 0 $\mathrm{h}$ de incubación fue evaluada por inmersión de las bolsas en agua a $40^{\circ} \mathrm{C}$ por una hora. En el tiempo predefinido, las bolsas fueron extraídas del rumen, y lavadas inmediatamente con agua fría en una lavadora 
comercial, durante un ciclo completo de lavado para remover la contaminación microbial en los residuos contenidos en las bolsas de nylon y luego de secadas a $105^{\circ} \mathrm{C}$ y pesadas individualmente, se unieron los residuos de las observaciones predeterminadas en cada repetición, preparadas para los análisis de materia seca, proteína cruda, extracto etéreo, fibra cruda y cenizas (AOAC 2002), así como fibra neutro detergente (Goering y Van Soest 1970). Con los valores residuales de materia seca, proteína y grasa, se determinó la cinética de la degradación usando el procedimiento descrito por Orskov y McDonald (1979). Los datos fueron ajustados a la ecuación exponencial:

$P=a+b\left(1-e^{-c t}\right)$,

donde $\mathrm{P}=$ degradación después de $\mathrm{t}$ horas de incubación ruminal

$\mathrm{a}=$ fracción soluble, $\%$

$\mathrm{b}=$ fracción degradable, $\%$

$\mathrm{c}=$ tasa de degradación, $\% / \mathrm{h}$

$\mathrm{t}=$ tiempo de incubación ruminal, $\mathrm{h}$.

Las constantes fueron calculadas por un programa de cuadrados mínimos iterativo, usando el algoritmo de Marquardt (Draper y Smith 1966).

Los valores de energía neta fueron estimados siguiendo los procedimientos del NRC (2001) para forrajes, usando el método biológico para determinar los valores de digestibilidad verdadera, ajustados para proteína y grasa a 48 horas de incubación ruminal, recomendado por Yu et al. (2004). Los métodos para determinar el total de nutrientes digestibles a una vez mantenimiento, así como la energía digestible y la neta para producción, mantenimiento y crecimiento, fueron los siguientes:

1.- $\mathrm{dvCNF}=0,98 \times\left\{100-\left[\left(\mathrm{FND}-\mathrm{PC}_{\mathrm{IFND}}\right)+\mathrm{PC}+\mathrm{EE}+\right.\right.$ donde:

Cen] $\} * \mathrm{~F}_{\mathrm{AP}}$

$\mathrm{dvCNF}=$ carbohidratos no fibrosos verdaderamente digestibles

$\mathrm{FND}=$ fibra neutro detergente

$\mathrm{PC}_{\mathrm{IFND}}=$ proteína cruda incrustada en la fibra neutro detergente

$\mathrm{PC}=$ proteína cruda en el forraje

$\mathrm{EE}=$ extracto etéreo en el forraje

Cen $=$ cenizas totales en el forraje
$\mathrm{F}_{\mathrm{AP}}=$ Factor de ajuste por procesamiento $=1$

2.- $\quad \mathrm{dvPC}=\mathrm{PC}_{48 \mathrm{H}} \times \mathrm{PC} \quad$ (método biológico)

donde:

$\mathrm{dvPC}=$ proteína cruda verdaderamente digestible

$\mathrm{PC}=$ proteína cruda en el alimento

$\mathrm{PC}_{\mathrm{D} 48 \mathrm{H}}=$ degradabilidad ruminal de la proteína cruda a 48 horas de incubación ruminal

3.- $\quad \mathrm{dvAG}=\mathrm{EE}_{48 \mathrm{H}} \times \mathrm{EE} \quad$ (método biológico)

donde: $\mathrm{EE}_{48 \mathrm{H}}=$ degradabilidad ruminal del extracto etéreo a 48 horas de incubación ruminal

4.- $\quad \operatorname{dvFND}=\left(\mathrm{FND}_{\mathrm{D} 48 \mathrm{H}} \times \mathrm{FND}-\mathrm{PC}_{\mathrm{IFND} 48 \mathrm{H}}\right)$

donde:

$\mathrm{dvFND}=$ fibra neutro detergente verdaderamente digestible $\mathrm{FND}_{\mathrm{D} 48 \mathrm{H}}=$ degradabilidad ruminal de la fibra neutro detergente a 48 horas de incubación ruminal

$\mathrm{PC}_{\mathrm{IFND48H}}=$ proteína cruda incrustada en la fibra neutro detergente

5.- $\mathrm{TND}_{1 \mathrm{X}}=\mathrm{dvCNF}+\mathrm{dvPC}+\mathrm{dvAG} \times 2,25+\mathrm{dvFND}-7$ donde:

$\mathrm{TND}_{1 \mathrm{X}}=$ total de nutrientes digestibles a una vez mantenimiento.

6.- $\mathrm{ED}_{1 \mathrm{x}}=\mathrm{dvCNF} \times 4,2 / 100+\mathrm{dvFND} \times 4,2 / 100+$ dvPC x 5,6/100+dvAG x 9,4/ $100-0,3$

donde:

$\mathrm{ED}_{1 \mathrm{X}}=$ energía digestible a una vez mantenimiento.

6.- $\mathrm{FD}=\left[\mathrm{TND}_{1 \mathrm{X} \%}-\left(0,18 \mathrm{x} \quad \mathrm{TND}_{1 \mathrm{X} \%}-10,3\right) \mathrm{x}\right.$ ConsTND $\left._{\mathrm{nX}-1}\right] / \mathrm{TND}_{1 \mathrm{X}} \%$

donde:

$\mathrm{FD}=$ factor de descuento, en tanto por 1 (de 0 a 1 )

$\mathrm{TND}_{1 \mathrm{X} \%}=$ concentración de TND en la dieta total, $\%$

ConsTND = consumo de TND sobre 1 vez mantenimiento

7.- $\mathrm{ED}_{\mathrm{P}}=\mathrm{ED}_{1 \mathrm{X}} \times \mathrm{FD}$

donde:

$\mathrm{ED}_{\mathrm{P}}=$ energía digestible a consumo actual de producción, $\mathrm{Mcal} / \mathrm{kg}$

8.- $\quad \mathrm{EM}_{\mathrm{P}}=1,01 \times \mathrm{ED}_{\mathrm{P}}-0,45$

donde:

$\mathrm{EM}_{\mathrm{P}}=$ energía metabolizable para producción

9.- $\quad \mathrm{EN}_{\mathrm{P}}=0,703 \times \mathrm{EM}_{\mathrm{P}}-0,19$

donde:

$\mathrm{EN}_{\mathrm{P}}=$ energía neta para producción, arriba de una vez mantenimiento. 
Para estimar la energía neta de mantenimiento y de crecimiento se usó la fórmula de energía metabolizable (EM) empleada por Garrett (1980), así:

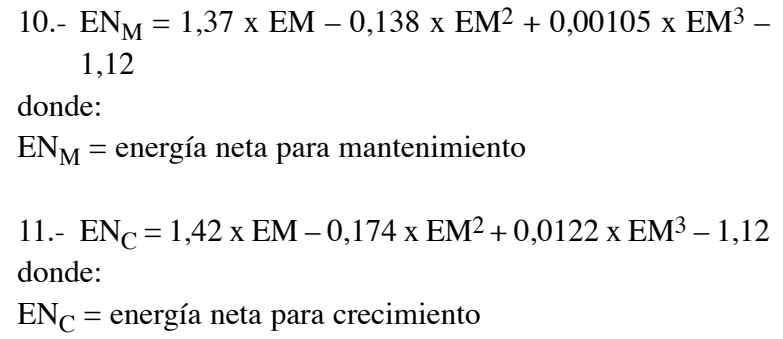

Las fracciones de proteína se determinaron biológicamente empleando los valores de degradación ruminal a 0 horas, como fracción A (proteína soluble) y el residuo proteico como fracción $\mathrm{C}$ (proteína indegradable) a 48 horas. La fracción $B$ (proteína degradable) se estimó como la proteína total menos la fracción A y la fracción C. En la determinación de la proteína degradada en el rumen se empleó la tasa de degradación encontrada en el ajuste de los datos al modelo de Orskov (1984), en cada edad de rebrote, con unidades de porcentaje por hora. La velocidad de pasaje fue estimada con base en el consumo de materia seca, peso vivo porcentual, para dos vacas típicas equivalentes, una Jersey de $400 \mathrm{~kg}$ de peso vivo con $20 \mathrm{~kg} /$ día de producción de leche y otra Holstein de $550 \mathrm{~kg}$ con $25 \mathrm{~kg}$ /día, respectivamente. Las mismas variables fueron empleadas en la determinación de proteína indegradable en el rumen y la fracción C encontrada en el residuo de la materia seca a 48 horas de incubación ruminal.

Los resultados de digestibilidad verdadera de los carbohidratos no fibrosos, proteína cruda, grasa, fibra neutro detergente y los valores de energía contenidos en las cuatro edades de rebrote, así como los valores de las fracciones proteicas y las proporciones de proteína degradable e indegradable, fueron sometidos al análisis de varianza (Gill 1978). El análisis estadístico se realizó con el PROC GLM de SAS (SAS 1985). Con las diferencias que se presentaron entre las edades de rebrote, se realizó una separación de medias mediante la prueba de Duncan.

\section{RESULTADOS}

En el Cuadro 1 se presenta el análisis proximal de la morera en cuatro estados de crecimiento. Se presentaron diferencias $(\mathrm{P} \leq 0,05)$ entre los contenidos de los componentes nutricionales, con excepción $(\mathrm{P} \geq$ $0,05)$ del contenido de hemicelulosa en tallos, fibra neutro detergente y sus componentes, así como la proteína cruda incrustada en la pared celular de las hojas en hoja, a través de los cuatro estados de crecimiento. En la planta entera, la concentración de lignina y de proteína incrustada en la fibra neutro detergente, muestran similitudes $(P \geq 0,05)$ independientemente de la edad. Los contenidos de materia seca en tallo son ligeramente inferiores que en la hoja a los 70 días de crecimiento, similares a los 84 días y superiores en el tallos a partir de los 98 días, con valores en la planta entera de 17 a $22,3 \%$ en forma creciente entre los 70 y 112 días de rebrote. Los valores de proteína cruda, extracto etéreo, cenizas y fibras en el tallo fueron similares $(\mathrm{P} \geq$ $0,05)$ a los 70 y 84 días, y a partir de esa edad la proteína y el extracto etéreo decrece $(\mathrm{P} \leq 0,05)$, mientras que la concentración de pared celular se incrementa $(\mathrm{P} \leq$ $0,05)$. Similar comportamiento se observó en las hojas y en consecuencia en la planta entera.

En el Cuadro 2 se muestra la concentración de los nutrientes verdaderamente digeridos y los contenidos de energía presentes en la morera en las cuatro edades de crecimiento estudiadas. De las cuatro fuentes de energía utilizable por los rumiantes, los tallos mostraron la máxima concentración de carbohidratos no fibrosos a los 84 días de rebrote $(\mathrm{P} \leq 0,05)$, mientras la proteína cruda y los ácidos grasos fueron superiores $(\mathrm{P}$ $\leq 0,05)$ a los 70 y 84 días sobre las siguientes etapas de crecimiento. En las edades iniciales, el tallo mostró la menor concentración de fibra neutro detergente, la cual se incrementó significativamente $(\mathrm{P} \leq 0,05)$ de los 98 días en adelante. En las hojas, las concentraciones de carbohidratos no fibrosos fueron estadísticamente similares en las cuatro edades $(\mathrm{P}>0,05)$, mientras la proteína cruda y ácidos grasos, fueron máximos a los 70 días y decrecieron conforme avanzó la edad de rebrote $(\mathrm{P} \leq 0,05)$. La mayor concentración de total de nutrientes digestibles se observó a los 84 días en los tallos $(P \leq 0,05)$, mientras que fue similar $(\mathrm{P} \geq 0,05)$ en las hojas a través de las diferentes edades estudiadas. Los valores de energía digestible a una vez mantenimiento y de producción, de energía neta para mantenimiento, crecimiento y producción siguieron similar comportamiento que el total de nutrientes digestibles en tallos; sin embargo, en hojas mostraron una diferencia importante a los 70 días $(\mathrm{P} \leq$ 0,05), con una leve disminución a los 84 y 98 días 
Cuadro 1. Composición química de la morera en cuatro edades de rebrote. Estación Experimental Alfredo Volio Mata, Tres Ríos, Cartago, Costa Rica. 2004.

\begin{tabular}{|c|c|c|c|c|c|c|c|c|c|}
\hline \multirow{3}{*}{\multicolumn{2}{|c|}{$\begin{array}{l}\text { Partes de la planta } \\
\text { Componentes }\end{array}$}} & \multicolumn{8}{|c|}{ Edad (días) } \\
\hline & & \multicolumn{2}{|c|}{70} & \multicolumn{2}{|c|}{84} & \multicolumn{2}{|c|}{98} & \multicolumn{2}{|r|}{112} \\
\hline & & $\mathbf{X}$ & DS & $\mathbf{X}$ & DS & $\mathbf{X}$ & DS & $\mathbf{X}$ & DS \\
\hline \multicolumn{10}{|l|}{ Tallo } \\
\hline & Materia seca, $\%$ & 15,59 & $0,89 \mathrm{c}$ & 16,68 & $2,27 \mathrm{c}$ & 20,55 & $1,06 \mathrm{~b}$ & 23,20 & $1,35 \mathrm{a}$ \\
\hline & Proteína cruda, \% & 11,25 & $0,99 \mathrm{a}$ & 10,83 & $1,21 \mathrm{a}$ & 7,83 & $0,75 \mathrm{~b}$ & 7,10 & $1,07 \mathrm{~b}$ \\
\hline & Extracto etéreo, \% & 1,18 & $0,15 \mathrm{a}$ & 1,14 & $0,19 \mathrm{a}$ & 0,75 & $0,08 \mathrm{c}$ & 0,98 & $0,19 \mathrm{~b}$ \\
\hline & Fibra neutro detergente, $\%$ & 63,65 & $1,54 \mathrm{~b}$ & 63,72 & $2,38 \mathrm{~b}$ & 71,73 & $5,64 \mathrm{a}$ & 73,02 & $4,77 \mathrm{a}$ \\
\hline & Hemicelulosa, \% & 12,56 & 1,53 & 14,83 & 4,29 & 15,67 & 4,91 & 15,52 & 3,68 \\
\hline & Fibra ácida detergente, $\%$ & 51,09 & $1,50 \mathrm{~b}$ & 48,89 & $5,30 \mathrm{~b}$ & 56,06 & $3,98 \mathrm{a}$ & 57,50 & $3,93 \mathrm{a}$ \\
\hline & Celulosa, \% & 45,24 & $1,14 \mathrm{ab}$ & 43,11 & $5,26 \mathrm{c}$ & 49,06 & $3,65 \mathrm{ab}$ & 50,39 & $3,71 \mathrm{a}$ \\
\hline & Lignina, $\%$ & 5,67 & $0,55 \mathrm{~b}$ & 5,78 & $0,35 \mathrm{~b}$ & 7,00 & $0,57 \mathrm{a}$ & 7,11 & $0,54 \mathrm{a}$ \\
\hline & Cenizas, $\%$ & 10,72 & $0,65 \mathrm{a}$ & 7,84 & $0,78 \mathrm{~b}$ & 6,50 & $0,68 \mathrm{c}$ & 6,59 & $0,76 \mathrm{c}$ \\
\hline & Proteína cruda PC en FND, \% & 3,66 & $0,36 \mathrm{a}$ & 2,30 & $0,45 \mathrm{~b}$ & 2,26 & $0,59 \mathrm{~b}$ & 2,21 & $0,29 \mathrm{~b}$ \\
\hline & Proteína cruda PC en FAD, \% & 2,03 & $0,42 \mathrm{a}$ & 1,49 & $0,20 \mathrm{~b}$ & 1,57 & $0,23 \mathrm{~b}$ & 1,52 & $0,26 \mathrm{~b}$ \\
\hline \multicolumn{10}{|l|}{ Hoja } \\
\hline & Materia seca, $\%$ & 17,73 & $0,52 \mathrm{c}$ & 16,68 & $0,83 \mathrm{c}$ & 23,69 & $3,71 \mathrm{a}$ & 21,59 & $1,21 \mathrm{~b}$ \\
\hline & Proteína cruda, \% & 25,45 & $1,15 \mathrm{a}$ & 25,60 & $1,17 \mathrm{a}$ & 23,61 & $0,78 \mathrm{~b}$ & 21,85 & $1,25 \mathrm{c}$ \\
\hline & Extracto etéreo, \% & 2,29 & $0,29 \mathrm{a}$ & 2,03 & $0,23 \mathrm{ab}$ & 1,87 & $0,29 \mathrm{~b}$ & 2,27 & $0,28 \mathrm{a}$ \\
\hline & Fibra neutro detergente, FND $\%$ & 36,47 & 8,12 & 37,99 & 6,63 & 40,10 & 9,45 & 44,31 & 9,05 \\
\hline & Hemicelulosa, \% & 9,32 & $5,12 \mathrm{ab}$ & 7,19 & $6,89 \mathrm{~b}$ & 12,40 & $7,19 \mathrm{ab}$ & 15,60 & $9,35 \mathrm{a}$ \\
\hline & Fibra ácida detergente, FAD \% & 27,15 & 4,48 & 30,79 & 7,32 & 27,70 & 4,06 & 28,71 & 5,67 \\
\hline & Celulosa, \% & 21,70 & 1,73 & 23,99 & 6,17 & 21,88 & 4,18 & 23,24 & 4,38 \\
\hline & Lignina, $\%$ & 5,45 & 3,23 & 6,81 & 3,38 & 5,82 & 2,16 & 5,47 & 1,77 \\
\hline & Cenizas, $\%$ & 16,58 & $0,41 \mathrm{c}$ & 17,08 & $0,71 \mathrm{bc}$ & 17,47 & $1,35 \mathrm{~b}$ & 18,74 & $0,41 \mathrm{a}$ \\
\hline & Proteína cruda en FND, \% & 9,67 & 2,50 & 10,31 & 1,97 & 10,98 & 2,59 & 10,38 & 2,68 \\
\hline & Proteína cruda en FAD, \% & 4,42 & $0,96 \mathrm{~b}$ & 7,07 & $2,70 \mathrm{a}$ & 5,92 & $0,83 \mathrm{ab}$ & 5,11 & $0,81 \mathrm{a}$ \\
\hline \multicolumn{10}{|c|}{ Planta entera } \\
\hline & Materia seca, $\%$ & 16,98 & $0,55 \mathrm{~b}$ & 16,70 & $1,16 \mathrm{~b}$ & 22,33 & $2,14 \mathrm{a}$ & 22,29 & $1,05 \mathrm{a}$ \\
\hline & Proteína cruda, $\%$ & 20,88 & $1,03 \mathrm{a}$ & 19,74 & $1,54 \mathrm{a}$ & 17,12 & $1,02 \mathrm{~b}$ & 15,04 & $0,95 \mathrm{c}$ \\
\hline & Extracto etéreo, \% & 1,93 & $0,20 \mathrm{a}$ & 1,67 & $0,18 \mathrm{~b}$ & 1,42 & $0,22 \mathrm{c}$ & 1,67 & $0,20 \mathrm{~b}$ \\
\hline & Fibra neutro detergente, $\%$ & 45,32 & $5,59 \mathrm{c}$ & 48,13 & $4,66 \mathrm{bc}$ & 53,29 & $6,58 \mathrm{ab}$ & 57,54 & $5,56 \mathrm{a}$ \\
\hline & Hemicelulosa, \% & 10,45 & $3,30 \mathrm{~b}$ & 10,23 & $4,69 \mathrm{~b}$ & 13,91 & $4,94 \mathrm{ab}$ & 15,52 & $5,32 \mathrm{a}$ \\
\hline & Fibra ácida detergente, $\%$ & 34,87 & $3,01 \mathrm{~b}$ & 37,90 & $6,44 \mathrm{ab}$ & 39,39 & $3,88 \mathrm{ab}$ & 42,01 & $4,27 \mathrm{a}$ \\
\hline & Celulosa, \% & 29,32 & $1,37 \mathrm{~b}$ & 31,51 & $5,63 \mathrm{~b}$ & 33,03 & $3,99 \mathrm{ab}$ & 35,79 & $3,70 \mathrm{a}$ \\
\hline & Lignina, $\%$ & 5,55 & 2,37 & 6,40 & 2,02 & 6,36 & 1,40 & 6,23 & 0,88 \\
\hline & Cenizas, \% & 14,70 & $0,49 \mathrm{a}$ & 13,42 & $0,95 \mathrm{~b}$ & 12,96 & $0,96 \mathrm{~b}$ & 13,13 & $0,43 \mathrm{~b}$ \\
\hline & Proteína cruda en FND, \% & 7,75 & 1,76 & 7,16 & 1,38 & 7,43 & 2,01 & 6,61 & 1,50 \\
\hline & Proteína cruda en FAD, \% & 3,64 & $0,59 \mathrm{~b}$ & 4,03 & $1,50 \mathrm{a}$ & 4,13 & $0,56 \mathrm{ab}$ & 3,46 & $0,43 \mathrm{~b}$ \\
\hline
\end{tabular}

$\mathrm{a}, \mathrm{b}, \mathrm{c}$ en una misma fila, marcan diferencias significativas $\mathrm{P}<0,05$

$(\mathrm{P} \geq 0,05)$, para nuevamente decrecer ligeramente a los 112 días. La planta entera sigue un patrón similar al observado en las hojas.

En el Cuadro 3 se presenta la degradabilidad ruminal de las proteína, así como las fracciones proteicas y las proporciones de proteína degradable e indegradable en rumen. Los valores de degradabilidad ruminal de la proteína cruda muestran que la fracción soluble en tallos es al menos el doble que en hojas y en ambos casos, máxima a los 70 días $(\mathrm{P} \leq 0,05)$ y ligeramente decreciente conforme avanzó la edad del rebrote. La fracción potencialmente degradable en rumen fue máxima a los 70 días $(\mathrm{P} \leq 0,05)$ en ambas partes de la planta, siendo en hojas al menos el tripe que en tallos. La velocidad de degradación en tallos fue 
Cuadro 2. Nutrientes verdaderamente digeridos y contenidos de energía de la morera en cuatro edades de rebrote. Estación Experimental Alfredo Volio Mata, Tres Ríos, Cartago, Costa Rica. 2004.

\begin{tabular}{|c|c|c|c|c|c|c|c|c|}
\hline \multirow{3}{*}{$\begin{array}{l}\text { Partes de la planta } \\
\text { Componentes }\end{array}$} & \multicolumn{8}{|c|}{ Edad (días) } \\
\hline & \multicolumn{2}{|c|}{70} & \multicolumn{2}{|c|}{84} & \multicolumn{2}{|c|}{98} & \multicolumn{2}{|c|}{112} \\
\hline & $\mathbf{X}$ & DS & $\mathbf{X}$ & DS & $\mathbf{X}$ & DS & $\mathbf{X}$ & DS \\
\hline \multicolumn{9}{|l|}{ Tallo } \\
\hline \multicolumn{9}{|l|}{ Digerido verdadero } \\
\hline Carbohidratos no fibrosos, $\%$ & 16,54 & $1,79 a b$ & 18,40 & $2,49 \mathrm{a}$ & 15,13 & $5,16 \mathrm{ab}$ & 14,24 & $3,24 \mathrm{~b}$ \\
\hline Proteína cruda, $\%$ & 9,06 & $0,78 \mathrm{a}$ & 9,19 & $1,21 \mathrm{a}$ & 6,15 & $0,74 \mathrm{~b}$ & 5,49 & $0,95 \mathrm{~b}$ \\
\hline Ácidos grasos, \% & 1,06 & $0,13 \mathrm{a}$ & 1,03 & $0,17 \mathrm{a}$ & 0,68 & $0,07 \mathrm{~b}$ & 0,88 & $0,16 \mathrm{~b}$ \\
\hline Fibra neutro detergente, $\%$ & 32,30 & $1,04 \mathrm{~b}$ & 33,11 & $1,69 \mathrm{~b}$ & 36,73 & $3,63 \mathrm{a}$ & 37,47 & $3,08 \mathrm{a}$ \\
\hline \multicolumn{9}{|l|}{ Energía } \\
\hline $\mathrm{TND}_{1 \mathrm{X}}, \%$ & 53,28 & $1,48 \mathrm{~b}$ & 56,00 & $0,63 \mathrm{a}$ & 52,54 & $2,17 \mathrm{~b}$ & 52,18 & $1,51 \mathrm{~b}$ \\
\hline $\mathrm{ED}_{1 \mathrm{X}}, \mathrm{mcal} / \mathrm{kg}$ & 2,36 & $0,07 \mathrm{~b}$ & 2,47 & $0,04 \mathrm{a}$ & 2,29 & $0,10 \mathrm{bc}$ & 2,26 & $0,08 \mathrm{c}$ \\
\hline $\mathrm{ED}_{\mathrm{P}}, \mathrm{mcal} / \mathrm{kg} / 1 / 2$ & 2,23 & $0,07 \mathrm{~b}$ & 2,34 & $0,03 \mathrm{a}$ & 2,16 & $0,09 \mathrm{bc}$ & 2,14 & $0,07 \mathrm{c}$ \\
\hline $\mathrm{EN}_{\mathrm{PL}}, \mathrm{mcal} / \mathrm{kg}$ & 1,07 & $0,05 \mathrm{~b}$ & 1,15 & $0,02 \mathrm{a}$ & 1,03 & $0,06 \mathrm{bc}$ & 1,01 & $0,05 \mathrm{c}$ \\
\hline $\mathrm{EN}_{\mathrm{M}}, \mathrm{mcal} / \mathrm{kg}$ & 1,09 & $0,07 \mathrm{~b}$ & 1,20 & 0,03 a & 1,02 & $0,09 \mathrm{bc}$ & 1,00 & $0,07 \mathrm{c}$ \\
\hline $\mathrm{EN}_{\mathrm{C}}, \mathrm{mcal} / \mathrm{kg}$ & 1,06 & $0,06 \mathrm{~b}$ & 1,16 & $0,03 \mathrm{a}$ & 1,00 & $0,08 \mathrm{bc}$ & 0,98 & $0,07 \mathrm{c}$ \\
\hline \multicolumn{9}{|l|}{ Hoja } \\
\hline \multicolumn{9}{|l|}{ Digerido verdadero } \\
\hline Carbohidratos no fibrosos, $\%$ & 28,30 & 5,42 & 27,06 & 5,26 & 27,37 & 7,44 & 22,75 & 7,44 \\
\hline Proteína cruda, $\%$ & 20,69 & $1,56 \mathrm{a}$ & 18,49 & $2,04 \mathrm{~b}$ & 17,49 & $0,67 \mathrm{bc}$ & 16,52 & $1,48 \mathrm{c}$ \\
\hline Ácidos grasos, \% & 2,06 & $0,26 \mathrm{a}$ & 1,83 & $0,21 \mathrm{ab}$ & 1,68 & $0,26 \mathrm{~b}$ & 2,04 & $0,25 \mathrm{a}$ \\
\hline Fibra neutro detergente, $\%$ & 10,71 & $2,12 \mathrm{~b}$ & 9,89 & $3,80 \mathrm{~b}$ & 11,67 & $4,21 \mathrm{ab}$ & 15,12 & $3,85 \mathrm{a}$ \\
\hline \multicolumn{9}{|l|}{ Energía } \\
\hline $\mathrm{TND}_{1 \mathrm{X}}, \%$ & 57,33 & 5,98 & 52,56 & 6,60 & 53,30 & 4,63 & 51,99 & 4,38 \\
\hline $\mathrm{ED} 1 \mathrm{X}, \mathrm{mcal} / \mathrm{kg}$ & 2,69 & $0,26 \mathrm{a}$ & 2,45 & $0,30 \mathrm{ab}$ & 2,48 & $0,19 \mathrm{ab}$ & 2,41 & $0,19 \mathrm{~b}$ \\
\hline $\mathrm{ED}_{\mathrm{P}}, \mathrm{mcal} / \mathrm{kg} / 1 / 2$ & 2,54 & $0,25 \mathrm{a}$ & 2,32 & $0,28 \mathrm{ab}$ & 2,34 & $0,18 \mathrm{ab}$ & 2,28 & $0,18 \mathrm{~b}$ \\
\hline $\mathrm{EN}_{\mathrm{PL}}, \mathrm{mcal} / \mathrm{kg}$ & 1,30 & $0,17 \mathrm{a}$ & 1,13 & $0,20 \mathrm{ab}$ & 1,16 & $0,13 \mathrm{ab}$ & 1,11 & $0,13 \mathrm{~b}$ \\
\hline $\mathrm{EN}_{\mathrm{M}}, \mathrm{mcal} / \mathrm{kg}$ & 1,39 & $0,24 \mathrm{a}$ & 1,18 & $0,28 \mathrm{ab}$ & 1,20 & $0,18 \mathrm{ab}$ & 1,13 & $0,19 \mathrm{~b}$ \\
\hline $\mathrm{EN}_{\mathrm{C}}, \mathrm{mcal} / \mathrm{kg}$ & 1,34 & $0,22 \mathrm{a}$ & 1,14 & $0,26 \mathrm{ab}$ & 1,16 & $0,17 \mathrm{ab}$ & 1,10 & $0,17 \mathrm{~b}$ \\
\hline \multicolumn{9}{|l|}{ Planta entera } \\
\hline \multicolumn{9}{|l|}{ Digerido verdadero } \\
\hline Carbohidratos no fibrosos, $\%$ & 24,43 & $3,85 \mathrm{a}$ & 23,72 & $3,67 \mathrm{a}$ & 22,20 & $5,61 \mathrm{ab}$ & 18,85 & $4,38 \mathrm{~b}$ \\
\hline Proteína cruda, $\%$ & 16,95 & $1,35 \mathrm{a}$ & 14,77 & $1,76 \mathrm{~b}$ & 12,82 & $0,82 \mathrm{c}$ & 11,42 & $1,07 \mathrm{~d}$ \\
\hline Ácidos grasos, $\%$ & 1,73 & $0,17 \mathrm{a}$ & 1,51 & $0,16 \mathrm{~b}$ & 1,28 & $0,20 \mathrm{c}$ & 1,51 & $0,17 \mathrm{~b}$ \\
\hline Fibra neutro detergente, $\%$ & 17,45 & $1,69 \mathrm{c}$ & 18,54 & $2,93 \mathrm{c}$ & 21,78 & $4,06 \mathrm{~b}$ & 25,29 & $2,44 \mathrm{a}$ \\
\hline \multicolumn{9}{|l|}{ Energía } \\
\hline $\mathrm{TND}_{1 \mathrm{X}}, \%$ & 55,73 & 4,57 & 53,42 & 4,38 & 52,67 & 3,26 & 51,73 & 0,18 \\
\hline $\mathrm{ED}_{1 \mathrm{X}}, \mathrm{mcal} / \mathrm{kg}$ & 2,57 & $0,20 \mathrm{a}$ & 2,46 & $0,20 \mathrm{ab}$ & 2,39 & $0,13 \mathrm{~b}$ & 2,34 & $0,12 \mathrm{~b}$ \\
\hline $\mathrm{ED}_{\mathrm{P}}, \mathrm{mcal} / \mathrm{kg} / 1 / 2$ & 2,43 & $0,19 \mathrm{a}$ & 2,31 & $0,18 \mathrm{ab}$ & 2,25 & $0,12 \mathrm{ab}$ & 2,21 & $0,11 \mathrm{~b}$ \\
\hline $\mathrm{EN}_{\mathrm{PL}}, \mathrm{mcal} / \mathrm{kg}$ & 1,22 & $0,13 \mathrm{a}$ & 1,14 & $0,13 \mathrm{ab}$ & 1,09 & $0,09 \mathrm{ab}$ & 1,06 & $0,08 \mathrm{~b}$ \\
\hline $\mathrm{EN}_{\mathrm{M}}, \mathrm{mcal} / \mathrm{kg}$ & 1,29 & $0,19 \mathrm{a}$ & 1,19 & $0,19 \mathrm{ab}$ & 1,11 & $0,13 \mathrm{ab}$ & 1,06 & $0,11 \mathrm{~b}$ \\
\hline $\mathrm{EN}_{\mathrm{C}}, \mathrm{mcal} / \mathrm{kg}$ & 1,25 & $0,17 \mathrm{a}$ & 1,15 & $0,17 \mathrm{ab}$ & 1,09 & $0,12 \mathrm{ab}$ & 1,04 & $0,11 \mathrm{~b}$ \\
\hline
\end{tabular}

a, b, c, d en una misma fila, marcan diferencias significativas $\mathrm{P}<0,05$.

$\mathrm{TND}_{1 \mathrm{X}}$ Total de nutrientes digestibles; $\mathrm{ED}_{1 \mathrm{X}}$ Energía digestible a una vez mantenimiento; $\mathrm{ED}_{\mathrm{P}}$ Energía digestible a nivel de producción; $\mathrm{EN}_{\mathrm{PL}}$ Energía neta para producción de leche; $\mathrm{EN}_{\mathrm{M}}$ Energía neta para mantenimiento; ENC Energía neta para crecimiento. $/ 1$ Factor de descuento 0,945 , para vacas Jersey de $400 \mathrm{~kg}$ y $20 \mathrm{~kg} /$ día o vacas Holstein de $550 \mathrm{~kg}$ de peso vivo y $25 \mathrm{~kg} /$ día de producción de leche.

12 Consumo a tres veces mantenimiento. 
Cuadro 3. Degradabilidad ruminal de la proteína, fracciones proteicas y proporción de proteína, degradable en el rumen. Estación Experimental Alfredo Volio Mata, Tres Ríos, Cartago, Costa Rica. 2004.

\begin{tabular}{|c|c|c|c|c|c|c|c|c|}
\hline \multirow{3}{*}{$\begin{array}{l}\text { Partes de la planta } \\
\text { Componentes }\end{array}$} & \multicolumn{8}{|c|}{ Edad (días) } \\
\hline & \multicolumn{2}{|c|}{70} & \multicolumn{2}{|c|}{84} & \multicolumn{2}{|c|}{98} & \multicolumn{2}{|r|}{112} \\
\hline & $\mathbf{X}$ & DS & $\mathbf{X}$ & DS & $\mathbf{X}$ & DS & $\mathbf{X}$ & DS \\
\hline \multicolumn{9}{|l|}{ Tallo } \\
\hline \multicolumn{9}{|l|}{ Degradabilidad ruminal } \\
\hline Fracción soluble (a), \% & 55,47 & $2,90 \mathrm{a}$ & 54,54 & $2,36 \mathrm{a}$ & 51,45 & $2,55 \mathrm{~b}$ & 48,35 & $2,28 \mathrm{c}$ \\
\hline Fracción potencial degradable (b), \% & 25,91 & $1,45 \mathrm{a}$ & 22,14 & $1,32 \mathrm{~b}$ & 21,66 & $1,35 \mathrm{~b}$ & 21,18 & $1,12 \mathrm{~b}$ \\
\hline Velocidad de degradación (c), \%/h & 13,16 & $1,01 \mathrm{a}$ & 14,43 & $1,13 \mathrm{a}$ & 13,87 & $1,12 \mathrm{a}$ & 13,32 & $1,19 \mathrm{a}$ \\
\hline Degradación a $48 \mathrm{~h}, \%$ & 81,34 & $2,08 \mathrm{a}$ & 76,66 & $2,02 \mathrm{~b}$ & 73,08 & $1,96 \mathrm{c}$ & 69,50 & $2,04 \mathrm{~d}$ \\
\hline \multicolumn{9}{|l|}{ Fracciones proteicas } \\
\hline Fracción soluble (A), \% & 55,47 & 2,90 & 54,54 & 2,36 & 51,45 & 2,55 & 48,35 & 2,28 \\
\hline Fracción degradable (B), \% & 42,50 & $0,42 \mathrm{~d}$ & 43,97 & $0,20 \mathrm{c}$ & 46,98 & $0,23 \mathrm{~b}$ & 50,13 & $0,26 \mathrm{a}$ \\
\hline Fracción indegradable $(\mathrm{C}), \% / 3$ & 2,03 & $0,42 \mathrm{a}$ & 1,49 & $0,20 \mathrm{~b}$ & 1,57 & $0,23 \mathrm{~b}$ & 1,52 & $0,26 \mathrm{~b}$ \\
\hline Proteína degradable en rumen, $\% / 1 / 2$ & 86,45 & $0,31 \mathrm{~b}$ & 87,36 & $0,15 \mathrm{a}$ & 86,17 & $0,17 \mathrm{c}$ & 85,00 & $0,19 \mathrm{~d}$ \\
\hline Proteína indegradable en rumen, $\% / 1 / 2$ & 13,55 & 0,31 & 12,64 & 0,15 & 13,83 & 0,17 & 15,00 & 0,19 \\
\hline \multicolumn{9}{|l|}{ Hoja } \\
\hline \multicolumn{9}{|l|}{ Degradabilidad ruminal } \\
\hline Fracción soluble (a), \% & 23,86 & $1,54 \mathrm{a}$ & 19,32 & $0,89 \mathrm{~b}$ & 18,03 & $0,72 \mathrm{~b}$ & 16,75 & $0,66 \mathrm{c}$ \\
\hline Fracción potencial degradable (b), \% & 75,03 & $2,78 \mathrm{~b}$ & 79,53 & $2,63 \mathrm{a}$ & 80,22 & $2,87 \mathrm{a}$ & 80,90 & $2,85 \mathrm{a}$ \\
\hline Velocidad de degradación (c), \%/h & 10,16 & $0,98 \mathrm{a}$ & 7,68 & $0,69 \mathrm{~b}$ & 6,94 & $0,54 \mathrm{c}$ & 6,21 & $0,71 \mathrm{c}$ \\
\hline Degradación a $48 \mathrm{~h}, \%$ & 98,32 & $2,12 \mathrm{a}$ & 96,86 & $1,81 \mathrm{ab}$ & 95,39 & $1,65 \mathrm{c}$ & 93,54 & $1,73 \mathrm{bc}$ \\
\hline \multicolumn{9}{|l|}{ Fracciones proteicas } \\
\hline Fracción soluble (A), \% & 23,86 & 1,54 & 19,32 & 0,89 & 18,03 & 0,72 & 16,75 & 0,66 \\
\hline Fracción degradable (B), \% & 71,72 & $0,96 \mathrm{~d}$ & 73,61 & $2,69 \mathrm{c}$ & 76,05 & $0,83 \mathrm{~b}$ & 78,14 & $0,81 \mathrm{a}$ \\
\hline Fracción indegradable $(\mathrm{C}), \% / 3$ & 4,42 & $0,96 \mathrm{~b}$ & 7,07 & $2,69 \mathrm{a}$ & 5,92 & $0,83 \mathrm{ab}$ & 5,11 & $0,81 \mathrm{~b}$ \\
\hline Proteína degradable en rumen, $\% / 1 / 2$ & 72,26 & $0,65 \mathrm{a}$ & 64,27 & $1,64 \mathrm{~b}$ & 62,63 & $0,49 \mathrm{c}$ & 60,43 & $0,45 \mathrm{~d}$ \\
\hline Proteína indegradable en rumen, $\% / 1 / 2$ & 27,74 & 0,65 & 35,73 & 0,65 & 37,37 & 0,65 & 39,57 & 0,65 \\
\hline \multicolumn{9}{|l|}{ Planta entera } \\
\hline \multicolumn{9}{|l|}{ Degradabilidad ruminal } \\
\hline Fracción soluble (a), \% & 37,43 & 1,76 & 35,74 & 1,64 & 35,17 & 1,42 & 34,61 & 1,85 \\
\hline Fracción potencial degradable (b), \% & 53,74 & $2,74 \mathrm{a}$ & 52,60 & $2,56 \mathrm{a}$ & 49,42 & $2,05 a b$ & 46,24 & $2,70 \mathrm{~b}$ \\
\hline Velocidad de degradación (c), \%/h & 10,63 & $1,01 \mathrm{a}$ & 8,87 & $0,85 \mathrm{~b}$ & 8,80 & $0,73 \mathrm{~b}$ & 8,72 & $0,89 \mathrm{~b}$ \\
\hline Degradación a 48h, \% & 90,84 & $1,89 \mathrm{a}$ & 87,60 & $1,86 \mathrm{a}$ & 83,87 & $1,54 \mathrm{~b}$ & 80,15 & $1,58 \mathrm{~b}$ \\
\hline \multicolumn{9}{|l|}{ Fracciones proteicas } \\
\hline Fracción soluble (A), \% & 37,43 & 1,76 & 35,74 & 1,64 & 35,17 & 1,42 & 34,61 & 1,85 \\
\hline Fracción degradable (B), \% & 58,93 & $0,59 \mathrm{c}$ & 59,43 & $1,49 \mathrm{c}$ & 60,70 & $0,56 \mathrm{~b}$ & 61,94 & $0,43 \mathrm{a}$ \\
\hline Fracción indegradable $(\mathrm{C}), \% / 3$ & 3,64 & $0,59 \mathrm{~b}$ & 4,83 & 1,49 a & 4,13 & $0,56 \mathrm{ab}$ & 3,45 & $0,42 \mathrm{~b}$ \\
\hline Proteína degradable en rumen, $\% / 1 / 2$ & 77,78 & $0,41 \mathrm{a}$ & 74,04 & $0,96 \mathrm{~b}$ & 74,17 & $0,36 \mathrm{~b}$ & 74,27 & $0,27 \mathrm{~b}$ \\
\hline Proteína indegradable en rumen, $\% / 1 / 2$ & 22,22 & 0,41 & 25,96 & 0,41 & 25,83 & 0,41 & 25,73 & 0,41 \\
\hline
\end{tabular}

a, b, c, d en una misma fila, marcan diferencias significativas $\mathrm{P}<0,05$.

$/ 1 \mathrm{Kd}$ igual a la velocidad de degradación (c) en \%/h.

$12 \mathrm{Kp}$ igual a 4,90\%/h, con un consumo de materia seca de $3 \%$ sobre el peso vivo.

13 Proteína dentro de la fibra ácido detergente.

similar $(P \geq 0,05)$ en todas las edades, mientras en hojas fue mayor $(\mathrm{P} \leq 0,05)$ a los 70 días, bastante menor a los 84 días, para ligeramente decrecer a los 98 y 112 días. La proporción de proteína degradable en rumen fue superior a $85 \%$ tallos y a $60 \%$ en hojas.

\section{DISCUSIÓN}

Al comparar la composición química de la morera a 70 días de crecimiento, con los resultados reportados por Kitahara et al. (2000) en Japón, se 
encuentran valores de proteína cruda similares de 23 a $25 \%$ en hojas, de $12,1 \%$ en tallos y $20,4 \%$ en la planta entera cortada a 60 días de crecimiento, indicando una composición de hojas y tallos similares en ambos casos, asimismo se reportaron contenidos de $31,6 \%$ de fibra neutro detergente en hojas y $60,5 \%$ en tallos, concordantes con las diferencias de edades indicadas. Sin embargo, en estudios realizados en El Salvador, Benavides et al. (2000) reportan valores superiores de proteína cruda, con niveles de $33,6 \%$ en las hojas superiores, $28,3 \%$ en las intermedias y $26,7 \%$ en las basales a una edad temprana de corte, así como extractos etéreos de $3,2 \%, 2,8 \%$, y $3,4 \%$ en las hojas superiores, mediales y basales, respectivamente. Valores similares de proteína cruda encontraron Martín et al. (2000) estudiando frecuencias de corte desde 45 hasta 90 días de rebrote, tanto en hojas como en tallos. Sánchez (2000) y Benavides et al. (2000) reportan en cabras digestibilidades in vivo de la materia seca de 78 a $81 \%$, de 80 a $89 \%$ en la hoja y de 37 a $44 \%$ en el tallo. Shayo (1997) reporta valores de $60 \%$ de digestibilidad en la cáscara del tallo, mientras Schmidek et al. (2000a) obtuvieron en Brasil valores de $23 \%$ de proteína cruda, $39 \%$ de fibra neutro detergente y $2,1 \%$ de extracto etéreo en las hojas de los rebrotes a 90 días, similares a las concentraciones del material en estudio. Independientemente de la localización geográfica de los cultivos de morera, la calidad bromatológica de la morera parece ser una característica constante.

Los resultados de degradabilidad observados en el presente experimento difieren con los encontrados en la literatura. Singh y Makkar (2000) en la India reportaron valores de $85 \%$ de degradabilidad potencial de la materia seca en morera madura y $52 \%$ de degradabilidad efectiva, con una tasa de degradación de 4,67\%/h. En proteína los valores fueron $95 \%$ de degradación potencial y $57 \%$ efectiva, con una velocidad de degradación ruminal de $6,72 \% / \mathrm{h}$. Almeida et al. (2000), en Brasil, reportaron en cabras digestibilidades entre 71 y $77 \%$ en la materia seca y de 76 a $78 \%$ en la proteína cruda, en edades de rebrote inferiores a 70 días. Benavides et al. (2000) reportaron valores de 3,7 Mcal de energía digestible/kg de materia seca. Así mismo, Schmidek et al. (2000a) han reportado concentraciones de 77 a $83 \%$ de nutrientes digestibles totales, equivalentes a una energía digestible de 3,5 a 3,6 Mcal/kg de materia seca, con niveles de proteína cruda, extracto etéreo y fibra neutro detergente similares a las hojas del rebrote a 70 días determinada en el presente estudio.
Los resultados de la degradación ruminal de las proteína encontrados en los tallos a los 70 días resultan altos, particularmente en tallos (55\% de fracción soluble), no así de $24 \%$ en hojas. Schmidek et al. (2000b) estudiando diferentes clones de morera encontraron en hojas valores de $24 \%$ de fracción soluble y $73 \%$ potencialmente degradable en rumen, similares a las observadas en el presente estudio en rebrotes de 70 días, pero con velocidades de degradación superiores de 15 a $18 \% / \mathrm{h}$. Es de notar que valores tan altos influyen en las proporciones de proteína degradable e indegradable en rumen. Schmidek et al. (2000a) reportaron una relación de 57 y $43 \%$, respectivamente. Los valores más cercanos en el presente estudio se encontraron a los 112 días en la proporción de 60,4 y 39,5\% de proteína degradable e indegradable en rumen, respectivamente. El NRC (2001) no tiene disponibles valores de proteínas digeribles e insolubles de morera que permitan comparar los resultados del presente estudio.

\section{CONCLUSIONES Y RECOMENDACIONES}

La morera mostró una composición química muy buena ( $>14 \%$ de proteína cruda y $<60 \%$ de fibra neutro detergente) a edades de 112 días de rebrote, siendo excelente ( $>17 \%$ de proteína cruda y $<55 \%$ de fibra neutro detergente) a menores edades. A mayor edad se perdió más aceleradamente el contenido de proteína en el rebrote del tallo que en las hojas. El contenido total de nutrientes digestibles, tanto en tallo como en hoja, fue prácticamente constante a través de las edades estudiadas (52-53\%), excepto a la edad de 70 días (57\%) en hoja. La concentración de energía neta para producción de leche observada en la planta entera se considera media (1,1-1,3 Mcal $\mathrm{EN}_{\mathrm{PL}} / \mathrm{kg}$ de materia seca en el follaje), mientras que la concentración de proteína fue alta $(>15 \%)$, de ella la fracción soluble es similar a una gramínea considerada excelente (25-30\%); sin embargo, la fracción degradable resultó alta $(>58 \%)$ y una fracción insoluble muy pequeña $(<5 \%)$. Estas características deben tenerse en cuenta a la hora de formular una mezcla total con alimentos ricos en energía y bajos en proteína con relación a la concentración deseada en la mezcla total. Por estas características se recomienda ofrecer la morera fraccionada, poniendo a disposición pequeñas cantidades varias veces al día. En trabajos 
subsiguientes, especialmente aquellos donde se mida el consumo, se recomienda evaluar la concentración de proteínas degradables en rumen y su consecuente efecto sobre el $\mathrm{pH}$ ruminal.

\section{LITERATURA CITADA}

ALMEIDA, J.E.; FONSECA, T.C. 2000. The high-trunk mulberry system in tropical climates. Mulberry for animal production. FAO Animal Production and Health, Paper 147:125-130.

AOAC (Association of Official Analysis Chemistry). 2002. Animal Feeds, Chapter 4. Washington D.C. USA. p. $1-6$.

BENAVIDES, J.; LACHAUX, M. ; FUENTES, M. 1994. Efecto de la aplicación de estiércol de cabra en el suelo sobre la calidad y producción de biomasa de morera (Morus, sp.). In: Benavides, J.E. ed. Árboles y arbustos forrajeros en América Central. Technical Report No. 67. Turrialba, Costa Rica, CATIE. p.495-514.

BENAVIDES, J.; HERNÁNDEZ, J.; ESQUIVEL, J.; VANCONCELOS, J.; GONZÁLEZ, J.; ESPINOSA, E. 2000. Supplementation of grazing dairy cattle with mulberry in Costa Rica. Mulberry for Animal Production. FAO Animal production and health, Paper 147:165-169.

BOSCHINI, C.; DORMOND, H.; CASTRO, A. 1999. Composición química de la morera (Morus alba), para uso en la alimentación animal: densidades y frecuencias de poda. Agronomía Mesoamericana 11:41-49.

BOSCHINI, C. 2003. Sustitución de alimento concentrado, con morera fresca (Morus alba), en la dieta de vacas lecheras. Agronomía Mesoamericana 14:185-192.

DRAPER, N. R; SMITH, H. 1966. Applied regression analysis . Wiley. New York, USA. 407 p.

GARRETT, W.N. 1980. Energy utilization by growing cattle as determined in 72 comparative slaughter experiments. Energy Metabolism Proceeding Symposium 26:3-7.

GILL, J.L. 1978. Design and analysis of experiments in the animal and medical sciences. Iowa State University Press. Volume 1. 409 p.
GOERING, H.K.; VAN SOEST, P.J. 1970. Forage fiber analysis (Apparatus, reagents, procedures and some applications). Agricultural Handbook No. American Research Service - United State - Department (ARSUS-DA), Washington, D.C., USA. 76 p.

KITAHARA, N.; SHIBATA, S.; NISHIDA, T. 2000. Management and utilization of mulberry for forage in Japan. 1. Productivity of the mulberry-pasture association system and nutritive value of mulberry. FAO Animal Production and Health, Paper 147:235-240.

MARTÍN, G.; REYES, F.; HERNÁNDEZ, I.; MILERA, M. 2000. Agronomic studies with mulberry in Cuba. 2000. FAO Animal Production and Health, Paper 147:103-113.

NRC (National Research Council). 1989. Nutrient requirements of dairy cattle. $6^{\text {th }}$ ed. National Academy Press. USA. 157 p.

NRC (National Research Council). 2001. Nutrient requirements of dairy cattle. 7 ed. National Academy Press. USA. $381 \mathrm{p}$.

ORSKOV, E.R. 1984. Evaluation of residues and agroindustrial by-products using the nylon bag method. FAO-ILCA Publication. Roma, Italia. 128 p.

ORSKOV, E.R., McDONALD. 1979. The estimation of protein degradability in the rumen from incubation measurements weighted according to the rate of passage. Journal of Agricultural Science 92 (499-503).

OVIEDO, J.F. 1995. Morera (Morus sp.) en asocio con Poró (Erithrina poeppigiana) y como suplemento para vacas lecheras en pastoreo. Tesis Magister Scientiae. Turrialba, Costa Rica, CATIE. 86 p.

SÁNCHEZ, M.D. 2000. Mulberry: an exceptional forage available almost worldwide. FAO Animal Production and Health, Paper 147:271-289.

SAS (Statistical Analysis System). 1985. User's guide: Statistics. 5 ed. SAS Institute Inc. Carry, North Carolina, USA. 57 p.

SAYO, C.M. 2000. The potential of mulberry as feed for ruminants in Central Tanzania. FAO Animal Production and Health, Paper 147:131-138. 